\title{
Design of Monitoring System Based on LabVIEW Producer/Consumer Model
}

\author{
Chunli Gao ${ }^{1}$, Jialin Zhu ${ }^{2}$ and Lusha Zhang ${ }^{3}$ \\ ${ }^{1}$ Beijing Information Science and Technology University, College of Automation, Beijing in China, 100192 \\ ${ }^{2}$ Beijing Information Science and Technology University, Electronic Information and Control Experimental Teaching Center, \\ Beijing in China, 100192 \\ ${ }^{3}$ Beijing Information Science and Technology University, College of Automation, Beijing in China, 100192
}

\begin{abstract}
In wireless monitoring systems, the program design mode has a great influence on data upload and processing efficiency. In order to solve the problem that data uploading and processing cannot be synchronized in the system, a LabVIEW system design based on the producer-consumer model is proposed. The data receiving unit, analysis processing unit and alarm unit are assigned to implement in three parallel cycles. The producer cycle is responsible for receiving the data transmitted by the wireless transmission module through the serial port, the two consumer cycles are responsible for data analysis and data extraction and filter for alarms and other functions. With this structure, the data can be collected, displayed and stored in real time. The experimental results show that this model increases the readability of the program and improves the efficiency of data uploading and processing.
\end{abstract}

Keywords-LabVIEW; producer-consumer model; parallel cycles

\section{INTRODUCTION}

LabVIEW is the most popular graphical virtual instrument software development environment introduced by National Instruments Corporation in the United States. The interface programming of LabVIEW software enables the analysis, processing and display functions of traditional instruments to be implemented by hardware and is replaced by powerful personal computers and monitors. The instrument function is completely realized by software programming[1]. Operation and application are simple and convenient. At the same time, LabVIEW is widely used at home and abroad. With the development and upgrading of LabVIEW, it is widely used in military, aerospace, power, machinery, construction and railway projects [2].

In wireless monitoring systems, the sequential mode is usually used, and the data is collected and processed. The process is troublesome, and it is also likely to be in error. Synchronization of data acquisition and data processing can be guaranteed by the queue in the LabVIEW Producer/Consumer mode, and it follows the principle of "first-in, first-out", that is, the data cyclically deposited by the producer into the queue will be taken out first in the consumer cycle, ensuring the reliability of data collection and processing. At the same time, the producer/consumer model is the master-slave loop. The main loop is executed in parallel with the slave loop. The data of the main loop is sent to the slave loop through the queue. Moreover, a producer multi-consumer structure has a more flexible and efficient processing mechanism and a stronger structural expansibility. Therefore, it is very meaningful to select a two-consumer cycle model of producer in LabVIEW to process the data in the wireless transmission module.

\section{Producer/CONSUMER Model}

\section{A. Concept}

The producer/consumer design model is the most basic design pattern in multi-threaded programming. It is a composite design pattern composed of a queue message processor and an event processor[3]. The design pattern is generally composed of cyclic structures and usually consisting of a producer loop structure and one or more consumer loop structures. The producer loop is the main loop, the consumer loop is the slave loop, and the data of the main loop is passed to the slave loop through the queue. If the main loop is not running, the slave loop does not run. And each cycle executes in parallel at different rates without affecting each other.

\section{B. Producer/Consumer Model Communication}

Synchronizing tasks and exchanging data between multiple VIs or between different threads of the same VI can be achieved by synchronous control techniques. The 'Synchronize' function palette is provided in LabVIEW, including notifiers, queues, semaphores, rendezvous, events, first call functions. And notifiers and queues usually implement data synchronization in the producer consumer mode. Both of the competition state problems can be well eliminated, but the queue has more buffers than the notifier, which can avoid data overwriting or loss and can store multiple data [3].

The queue in the producer/consumer model cycle is usually issued in a "first-in, first-out" manner. The first data uploaded by the wireless monitoring system through the serial port is placed at the end of the buffer, that is, at the end of the queue. When the consumer cycle is taken out, it will be taken out first, so that the data generated by the producer can be entered into the consumer cycle through the queue sequence for data analysis processing, etc. [4-6].Thus the communication of the producer cycle to the consumer cycle is completed.

\section{Producer/Consumer Model Structure}

In the producer/consumer model, the producer cycle and the consumer cycle are mainly included. The producer cycle uses the "elements into queue" function to add the uploaded data to the queue for recycling by the consumer. The consumer cycle 
uses the "element out queue" function to retrieve the data stored in the queue. If no elements can be extracted from the current queue, the function will wait until TIMEOUT or have data.

\section{VISA SERIAL PORT}

When the wireless monitoring system uploads data, the communication method is serial communication. Therefore, the VISA module is selected in LabVIEW. VISA is a general standard for instrument I/O. It has features that are independent of the instrument's hardware interface and specific computer. That is, VISA is device-oriented rather than interface-oriented. When VISA nodes are used for serial communication programming in LabVIEW, an additional NI-VISA driver is required. In this article, VISA configuration serial port, serial port writing, serial port reading, and serial port shutdown are mainly used. By using these modules, the communication parameters of the serial port can be configured and read from the data [7]. Among them, in the "VISA configuration serial port" module, according to the communication parameters of the lower computer, parameters such as baud rate, parity, stop bits, and flow control must be set. When using the serial read/write function, write data by "VISA write" function according to conditions, or read data by "VISA read" function [8]. Reading the data will always read the number of data specified by the reading value. The extra data will be read in the next cycle.

\section{PROGRAMMING}

\section{A. Overall Structure Introduction}

The transmission module in the wireless monitoring system uploads the received data to the PC through the serial port, and the producer creates a queue and stores the data in a queue buffer. Since the producer cycle and the consumer cycle run synchronously, when the producer cycle runs, the two parallel consumer cycles sequentially extract the queue buffer data for analysis and processing. At the same time, the alarm unit module sends out the relevant warning instruction of the maximum value of the data to achieve the purpose of monitoring the system. template is designed so that author affiliations are not repeated each time for multiple authors of the same affiliation. Please keep your affiliations as succinct as possible (for example, do not differentiate among departments of the same organization). This template was designed for two affiliations.

\section{B. Front Panel Design}

According to the functional requirements of the system, the front panel of the PC based on LabVIEW has functions such as graphic display, maximum transmission, historical data table, and clearing. The overall VI interface of the system is shown in Figure I.

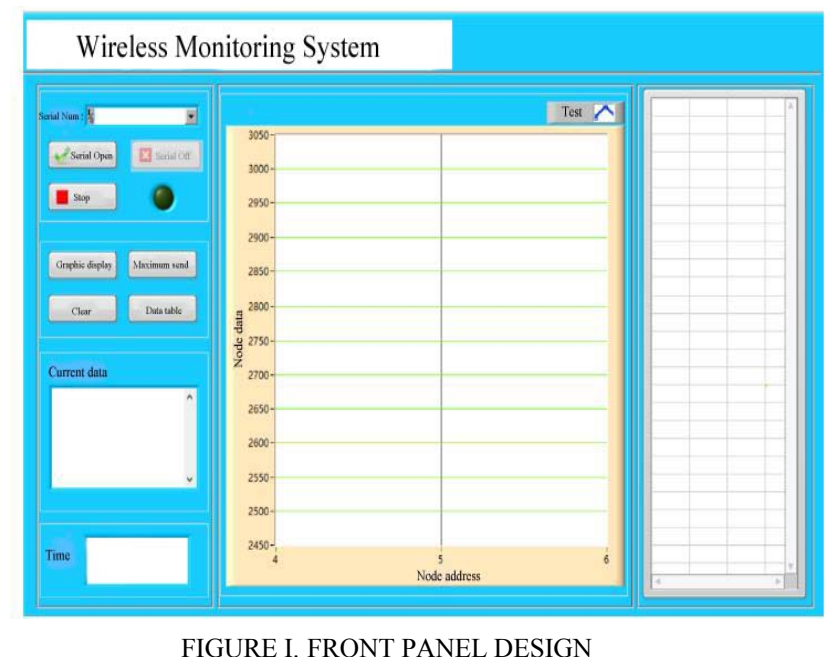

Because the upper computer and the wireless transmission module are connected through the serial port, the serial port of the wireless transmission module needs to be connected before the operation of "opening the serial port". It is necessary to set the communication parameters with the lower unit to be the same, including baud rate, parity, stop bits, and flow control. When data is uploaded, producer cycles and consumer cycles start their own work.

\section{Block Diagram Design}

\section{1) Serial port design unit:}

The serial port design unit is mainly for serial port initialization, that is to determine the serial port number, baud rate, parity, stop bits, flow control and other information. When the serial port number is used, it is selected according to the port prompt of the access terminal. When the serial port number is used, it is selected according to the port prompt of the access terminal. For the accurate transmission of the data from the wireless transmission module to the PC, the setting of the input port of the VISA function should be the same as the setting of the lower computer. The baud rate is uniformly set to $115200 \mathrm{bps}$, parity is none, the stop bit is $1 \mathrm{bit}$, and there is no flow control. This part also designed the serial port switch, serial port number selection and table. The design of the serial port requires the use of instrument $\mathrm{I} / \mathrm{O}-$-serial and programming-strings in the function palette. After the serial port number is selected, the serial port switch is turned on, and the collected data is stored in the corresponding position in the table.

\section{2) Data receiving unit:}

The data receiving unit module is equivalent to "producer". It mainly receives the monitoring data sent by the wireless transmission module. In the event structure, after the corresponding conditions are judged, the data is written with the "VISA write" function, and then the data is added to the queue after relevant calculations and judgments. The addition of a while loop allows producers to generate data and store it in queues. Click the "Open Serial Port" button, the serial port data enters the writing area and is stored in the corresponding location for further processing in the future. The block diagram is shown in Figure II. 


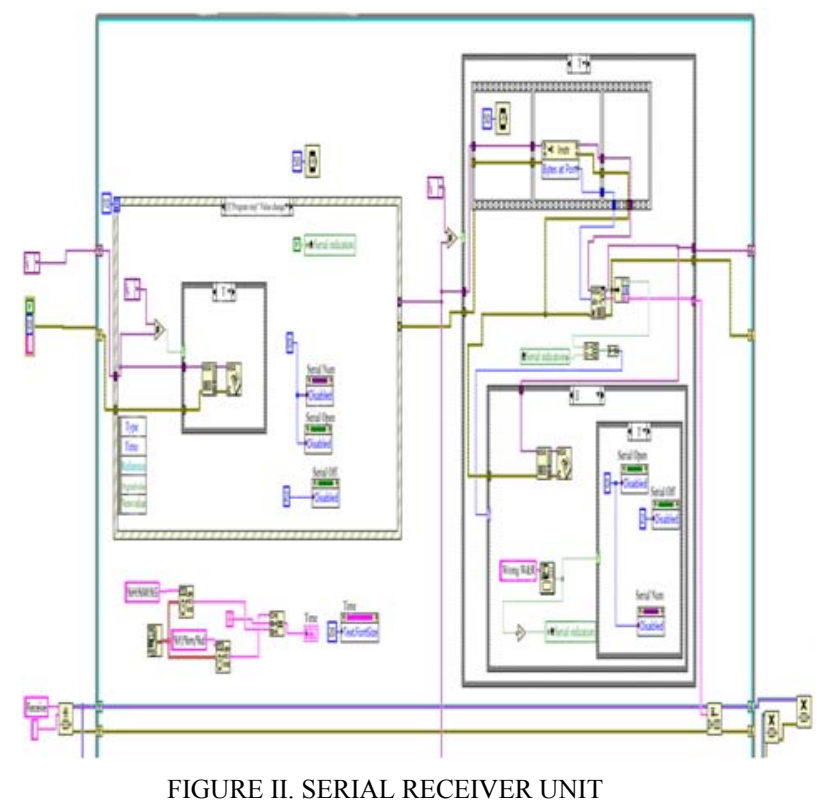

\section{3) Analysis and processing unit:}

The analysis and processing unit is equivalent to "consumer", that is, the entire module is a consumer cycle. In this structure, the conditional structure is nested to handle the data retrieved from the queue. The data will be displayed in the form of a waveform diagram to facilitate the user's intuitive access to relevant information. The unit block diagram is shown in Figure III.

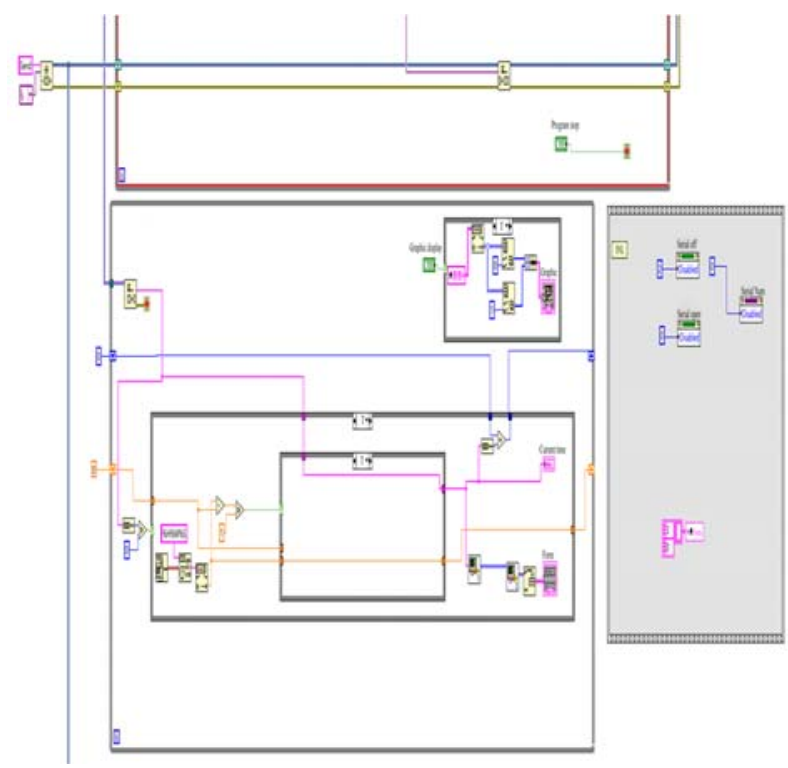

FIGURE III. ANALYSIS AND PROCESSING UNIT

\section{4) Alarm unit:}

In the wireless monitoring system, the PC needs to make feedback on the lower computer according to the data, and is called an alarm module. The alarm unit module is also a consumer cycle. It's similar to the analysis and processing unit program structure, the alarm unit module has an "element out queue" module whose function is to take out the data stored in the queue from the producer. The block diagram is shown in Figure IV.

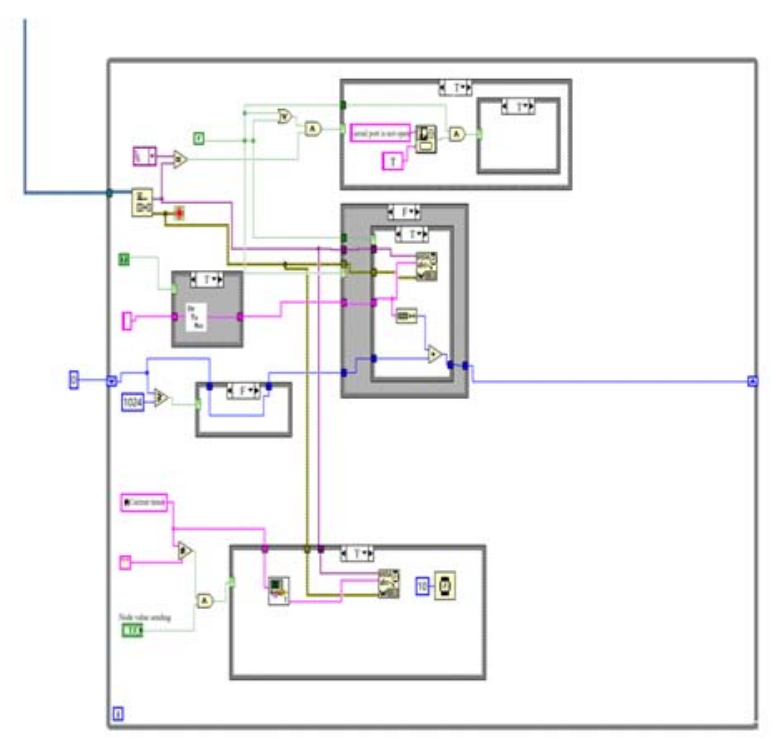

FIGURE IV. ALARM UNIT

During program execution, the producer cycle continuously generates data into the buffer, and the consumer cycle continuously reads data from the buffer. Because each cycle only performs its own work, there is no impact between the two. Producer cycles only generate data and the consumer cycle runs.

\section{CONCLUSION}

In this paper, the producer/consumer model is used to improve the operating efficiency of the program to a certain extent, and the problem of low efficiency and troublesome process of the sequential execution structure in the wireless monitoring system is solved. By introducing the structure of a producer cycle and two consumer cycles, the flexibility and efficiency of the model are demonstrated. At the same time, the LabVIEW programming control panel is used, the operation is simple, intuitive, and easy to use. It can transform complex and cumbersome language programming into a method of selecting functions by using dialog boxes or menu prompts. Each function module is connected by a line, which is simple and convenient, and saving program development time, and the operating speed is almost unaffected, with good humancomputer interaction and reliability.

\section{ACKNOWLEDGMENT}

I would like to thank all the students, brothers, sisters, and my roommates in the laboratory, especially Zhu, my life mentors. When I encounter academic problems, they always patiently help me and let me go further and further on my academic road. In particular, during the period when I first encountered LabVIEW programming, my sister always patiently guided me with Lusha Zhang. We discussed problems together and studied together, thanking them for giving me surprise and joy in my busy academic life.

Finally, I thank my parents and loved ones for their support 
and support for me over the years. Their selfless love is my driving force. Thanks for giving me encouragement and concern for teachers, classmates and friends in my career.

\section{REFERENCES}

[1] Yongqiang MA, Lin ZHOU. New Power Quality Real-time Monitoring System Based on LabVIEW[J].Electrical Measurement \& Instrumentation, 2009(03):40-44+71.

[2] Keman YANG. About Virtual Instrument and Its Application Prospects[J].Journal of Higher Correspondence (Natural Science), 2004(03):26-27+44

[3] Suzhen LIU, Yanjun WU, Hao ZHANG. Design of Stress Wave Information Acquisition System Based on Producer/Consumer Design Patterns[J].Computer Measurement \& Control, 2016, (9):198-202.

[4] Yiyao LEI, Zhi LI. Design of data acquisition system based on producer/consumer[J]. Computer Applications Technology, 2011, 9(38) :40 -45.

[5] Jian WANG, Yanjun ZHAI, Weiping WANG. Rail Contour Acquisition System Based on Producer/Consumer Model[J]. China Railways, 2014( 3):74 -76.

[6] Jinyuan TANG, Song LI, Wen SHAO. Geared Vibration Test System Based on LabVIEW Producer-Consumer Model[j]. Mechanical Transmission, 2011, 35 ( 1): 5- 7 .

[7] Datong QIN, Liyang XIE. Modern Mechanical Design Manual [M] Beijing: Chemical Industry Press, 2013: 216-218.

[8] Yangfan SHEN, Jianxin BAO, Xinwei ZHAO. LabVIEW-based workshop environment wireless monitoring system[J]. Instrument Technology and Sensors, 2017(09): 83-87. 